\title{
Stochastically generated turbulence for wall bounded flows
}

\author{
$\begin{array}{lll}\text { R. F. Jones } & \text { C. J. Doolan } & \text { M. D. Teubner } \\ & \end{array}$
}

(Received 22 March 2010; revised 14 July 2010)

\begin{abstract}
An efficient stochastic method is applied to the problem of modelling unsteady turbulent velocity fluctuations within a turbulent flat plate boundary layer. White noise is spatially and temporarily convoluted by the time averaged Reynolds stress tensor and dissipation. The convolutions are conducted such that mean turbulent properties are reproduced and the spectral distribution of turbulent kinetic energy closely matches expected profiles. Wall bounded turbulent flow induces elevated levels of broadband aeroacoustic noise, especially near a sharp edge such as an airfoil trailing edge. In practice, the stochastic method of generating turbulent fluctuations may be used directly to produce turbulent noise sources for further aeroacoustic analysis, thus avoiding costly time dependent simulations of the Navier-Stokes equations.
\end{abstract}

http://anziamj . austms.org.au/ojs/index.php/ANZIAMJ/article/view/2507 gives this article, (C) Austral. Mathematical Soc. 2010. Published August 12, 2010. IssN 1446-8735. (Print two pages per sheet of paper.) Copies of this article must not be made otherwise available on the internet, instead link direct to this URL for this article. 


\section{Contents}

1 Introduction

C542

2 Stochastic turbulence generation

C544

3 Computational results

C546

4 Conclusions

C548

References

C552

\section{Introduction}

Wall bounded turbulent flow induces elevated levels of broadband aeroacoustic noise, especially near a sharp edge such as an airfoil trailing edge. The success of traditional noise attenuation methods means these broadband noise sources are becoming increasingly important contributors to the overall level of noise generated by aircraft, wind turbines, submarines and cooling fans. By using time accurate large eddy simulation (LES) or direct numerical simulation (DNS) to compute near field turbulent sources of noise, a presumably accurate noise prediction can be made. However, for design purposes, these approaches are far too time consuming.

Computationally efficient and accurate methods of modelling wall bounded turbulence are needed in order to produce designs that reduce turbulent broadband noise at the source. Stochastic generation of turbulent velocities provides a computationally efficient method of obtaining time dependent turbulent fields which realise local statistical features. The stochastic method is based on spatial and temporal filtering of white noise. In practice, the stochastic techniques presented may be employed in combination with traditional Reynolds Averaged Navier-Stokes (RANS) methods, thus providing a 
computationally efficient approach to calculating noise source data for further aeroacoustic evaluation and design.

The stochastic method to generate turbulent velocities was first introduced by Kraichnan [13] based on random fourier modes. Improvements to the model have been conducted by Karweit et al. [11], Bechara et al. [2], Bailly and Juve [1], Blom et al. [4], Snellen et al. [18], Billson et al. [3] and Smirnov et al. [17] with varying degrees of complexity. These extensions primarily focussed on reducing computational effort and including time and convection effects. Of significance is the work by Billson et al. [3] to include an autocorrelation function to well mimic the Kolmogorov spectral scaling. Additionally, the work of Smirnov et al. [17] for the inclusion of anisotropic effects is significant; however, as noted by Mesbah [15], this method was developed as an inlet boundary condition for LES simulations and has not yet been used for aerocoustic analysis. The alternative to random fourier modes is digital filtering of white noise. Ewert [9, 10, 5, 6, 7, 8] used a digital filtering process originally proposed by Klein et al. [12] to generate turbulent velocities with a given spatial correlation. Ewert highlighted the transformations of Lund et al. [14] and Smirnov et al. [17] as methods which could be used for including anisotropic effects. Mesbah [15] proposed a combined method, whereby the methods of Ewert [9], Lund et al. [14] and Billson et al. [3] are used to generate a turbulence velocity field.

The quality of stochastically generated turbulence by the combined method of Mesbah is studied for wall bounded flows. There are a number of novel aspects to this work. Firstly, Mesbah [15] has not done a detailed comparison of boundary layer spectra with the stochastic method, and this is the first time it has been done. Secondly, this is the first time that anisotropic autocorrelation model coefficients are permitted, which allows for more accurate recreation of the anisotropic turbulent spectra in the boundary layer.

The technique is demonstrated for flow over a flat plate. Comparison is made to direct numerical simulations showing the ability of the stochastic method to recreate expected spectral distributions of turbulent kinetic energy. 


\section{Stochastic turbulence generation}

The methods presented closely follow those of Mesbah [15]. Accurate generation of stochastic turbulent velocity data requires an account of mean turbulence statistics and the anisotropic Reynolds stress tensor. White noise, uncorrelated in each direction, is spatially filtered by the approach of Ewert [9]. Anisotropic Reynolds stresses are accounted for by the Lund et al. [14] transformation and temporal scaling via the autocorrelation [3]. However, in the formulation used by Mesbah [15] the autocorrelation function differs from that originally proposed by Billson et al. [3]. In order to maintain consistency with previous work the original autocorrelation function of Billson et al. [3] is employed.

A vector is created of length $n$ of random numbers representing the velocity field in the ith direction for position $x, r_{i}^{n}(x)$ with zero mean, unit variance and zero covariance. This vector of random numbers is then manipulated to give the desired statistical characteristics. Initially, the Gaussian random velocity field $r(x)$ is filtered by the kernel $G(x)$ to give a velocity streamfunction $\psi$ with a prescribed length scale

$$
\psi(x)=\int_{-\infty}^{+\infty} G\left(x-x^{\prime}\right) \cdot r\left(x^{\prime}\right) d x^{\prime},
$$

where

$$
\mathrm{G}(\mathrm{x})=\mathrm{A} \exp \left(-\frac{\pi}{2} \frac{\mathrm{x}^{2}}{\mathrm{~L}^{2}}\right)
$$

such that $L$ is the integral length scale estimated from the steady turbulent statistical data. The integral indicates the influence of the turbulent velocity field at any $x^{\prime}$ location upon the velocity field at the $x$ location. Therefore separate integral length scales in the ith, $j$ th and kth directions are applied using

$$
\mathrm{G}(\mathrm{x})=\exp \left(-\frac{\pi}{2} \frac{x_{i}^{2}}{\mathrm{~L}_{\mathrm{i}}^{2}}\right) \exp \left(-\frac{\pi}{2} \frac{x_{j}^{2}}{\mathrm{~L}_{j}^{2}}\right) \exp \left(-\frac{\pi}{2} \frac{x_{k}^{2}}{\mathrm{~L}_{\mathrm{k}}^{2}}\right),
$$


where the integral length scale is approximated by

$$
\mathrm{L}_{\mathrm{i}}=\mathrm{c}_{l} \frac{\mathrm{R}_{\mathfrak{i i}}^{3 / 2}}{\epsilon},
$$

where $R_{i i}$ is the mean Reynolds stress in the ith direction, $\epsilon$ is the mean dissipation, and $c_{l}=0.54$ for a modified Von Karman turbulence energy spectrum [1]. The coefficient $c_{l}$ may be used as a tuning variable in order to tailor the stochastic response to a particular problem, and may vary up to a value of 1 . This procedure was adopted by Ewert [8], who found that a value of 0.675 was most appropriate for matching turbulent trailing edge predictions to experimental results.

The turbulent velocity field is then scaled by the mean anisotropic Reynolds stress tensor using the method of Klein et al. [12]:

$$
v_{i}^{\prime n}=\Gamma_{i j} \psi_{j}^{n},
$$

where

$$
\Gamma_{i j}=\left[\begin{array}{ccc}
\sqrt{R_{11}} & 0 & 0 \\
R_{21} / \Gamma_{11} & \sqrt{R_{22}-\Gamma_{21}^{2}} & 0 \\
R_{31} / \Gamma_{11} & \left(R_{32}-\Gamma_{21} \Gamma_{31}\right) / \Gamma_{22} & \sqrt{R_{22}-\Gamma_{31}^{2}-\Gamma_{32}^{2}}
\end{array}\right],
$$

where $R_{i j}$ is the mean Reynolds stress component ( $i j$ ). An additional scaling matrix is included here for adjusting the magnitude of the resultant turbulent kinetic energy. This scaling is applied to each velocity direction by premultiplying $\Gamma$ by the matrix

$$
\mathrm{m}_{\Gamma}=\left[\begin{array}{ccc}
\mathrm{m}_{1} & 0 & 0 \\
0 & \mathrm{~m}_{2} & 0 \\
0 & 0 & \mathrm{~m}_{3}
\end{array}\right] .
$$

The time scale is then included using the method of Billson et al. [3]. This renders the final vector, $\mathfrak{u}_{i}^{\prime}$ of turbulent velocity fluctuations in the ith direction as

$$
u_{i}^{\prime n}=a u_{i}^{\prime n-1}+b\left(v_{i}^{\prime n}+v_{i}^{\prime n-1}\right),
$$


where

$$
a=\exp \left[1 /\left(\tau F_{s}\right)\right] \text { and } b=\sqrt{\left(1-a^{2}\right) / 2},
$$

where $F_{s}$ is an assumed sampling frequency, and the time scale $\tau=f_{\tau} \overline{k_{e}} / \epsilon$ is calculated from the RANS solution by the ratio of total turbulent kinetic energy $k_{e}$ to dissipation $\epsilon . f_{\tau}$ is a tuning parameter for adjusting the time scale response to better represent experimental data.

While not explicitly stated by either Billson et al. [3] or Mesbah [15], it is implied that a single instance of $a$ and $b$ are used to filter the stochastic velocity fluctuations irrespective of the direction of motion $i$. We allow different values of $a$ and $b$ in different directions by making allowance for three autocorrelation tuning parameters which are now be defined as $\left(f_{\tau}\right)_{i}$. This has the effect of allowing the spectral shape to be tailored to the expected spectral distribution for a particular turbulent velocity component.

\section{Computational results}

The turbulent, flat plate boundary layer DNS data of Spalart [19] are used for comparison with the stochastically generated data created using the method described in Section 2. The Spalart [19] mean statistical data is available from the ERCOFTAC classic collection database ${ }^{1}$. The highest Reynolds number case of $\operatorname{Re}=1410$ (based on boundary layer momentum thickness) was chosen since low Reynolds number effects are minimized and the turbulent fluctuations show a significant inertial range [19]. These are Reynolds number conditions expected in the intended application to external boundary layer noise from airfoils.

Data available for download consists of mean turbulent properties and velocities at a number of vertical stations. Spectra from Spalart [19] are reported at locations where $y+=100$ and 200 and are normalized by a variety of

\footnotetext{
${ }^{1}$ http://cfd.mace.manchester.ac.uk/ercoftac/
} 
scales according to the approach of Perry et al. [16]. Unfortunately, a number of these scales are not available. For the current work, the Kolmogorov velocity $\mathfrak{u}_{k}$ and length $l_{k}$ scales were deemed to be the most easily estimated since they may be calculated from the given mean turbulence statistics

$$
u_{k}=(v \epsilon)^{1 / 4}, \quad l_{k}=\left(v^{3} / \epsilon\right)^{1 / 4}
$$

where $v$ is the kinematic viscosity. To calculate stochastic velocity spectra the frequency limits were first set according to the frequency limits of the Spalart [19] comparison data. The maximum wavenumber was set by the sampling frequency $F_{s}$ and the lowest wavenumber by the length $n$ of the randomly generated vector, $r$. The Gamma matrix of equation (6) was then populated by the mean turbulent energy values. Here we assumed that $\Gamma_{31}=\Gamma_{32}=0$ since the required values were not available. However, for flat plate boundary layer flow these terms were expected to be a number of orders of magnitude smaller than the $\Gamma_{33}$ term and therefore insignificant. The fourier transform of the velocity field was then calculated using a Hamming window and the spectra was averaged over 3000 samples to give the spectral results presented below. Evaluation time for these settings were under 15 seconds using Matlab on a quadcore Intel Core $2.4 \mathrm{GHz}$ machine with $4 \mathrm{~GB}$ RAM. The $m_{i}$ and $\left(f_{\tau}\right)_{i}$ were then adjusted to match the spectral magnitude and shape of Spalart [19]. The final stochastic turbulent energy spectra E and wavenumbers $k$ results are scaled by the Kolmogorov parameters and compared with Spalart [19] in Figures 1 through 6. For the $y+=100$ case the tuning parameters were set to

$$
\begin{aligned}
& m_{1}=0.33, \quad m_{2}=0.5, \quad m_{3}=0.5, \\
& f_{\tau}(u)=1.8 \times 10^{-6}, \quad f_{\tau}(v)=0.5 \times 10^{-6}, \quad f_{\tau}(w)=1.5 \times 10^{-6},
\end{aligned}
$$

while the only adjustment required for $y+=200$ was to set $m_{1}=0.53$. The results seem to match the DNS data very well, with some discrepancies at the lowest and highest wavenumbers. Also, the $w$ velocity spectra seems to be over represented at high wavenumbers by a much larger amount than other velocity components. This is primarily a result of the autocorrelation 


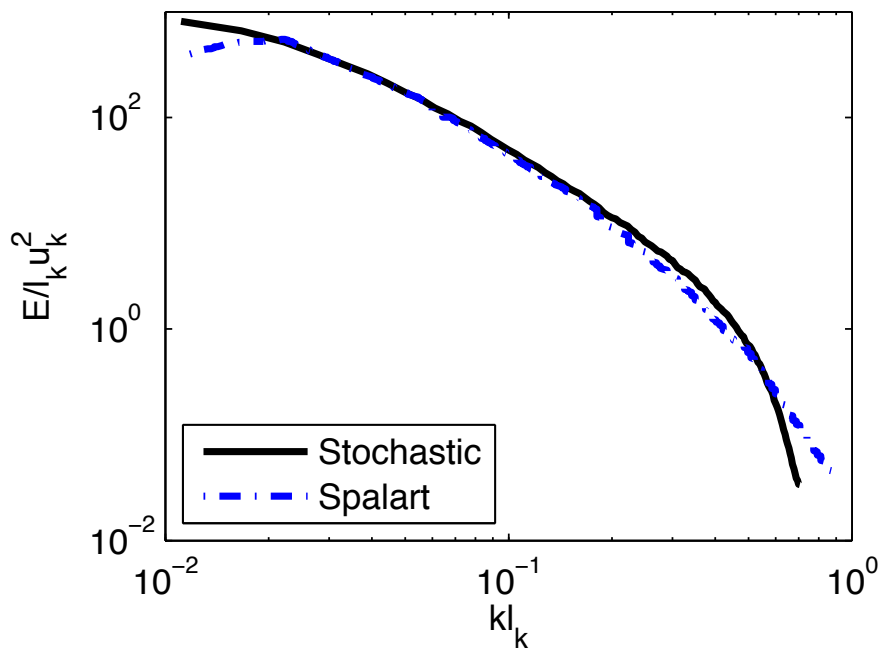

FiguRE 1: Turbulent kinetic energy spectra at $y+=100$ corresponding to the $u$ velocity component. Stochastic results using the current method are compared with results of Spalart [19]. Kolmogorov velocity and length scales are $\mathfrak{u}_{\mathrm{k}}=0.0290$ and $\boldsymbol{l}_{\mathrm{k}}=5.1733 \times 10^{-4}$ respectively.

formulation for the $a$ and $b$ values which sets the slope of the spectra before the high wavenumber roll-off.

\section{Conclusions}

An efficient stochastic method has been applied to the problem of modelling unsteady turbulent velocity fluctuations within a turbulent flat plate boundary layer. White noise is spatially and temporally convoluted by the time averaged Reynolds stress tensor and dissipation. The convolutions were conducted such that the spectral distribution of turbulent kinetic energy closely matches expected profiles. We found that while some discrepancies exist at the lowest and highest wavenumbers, the stochastic method recreated turbulent energy 


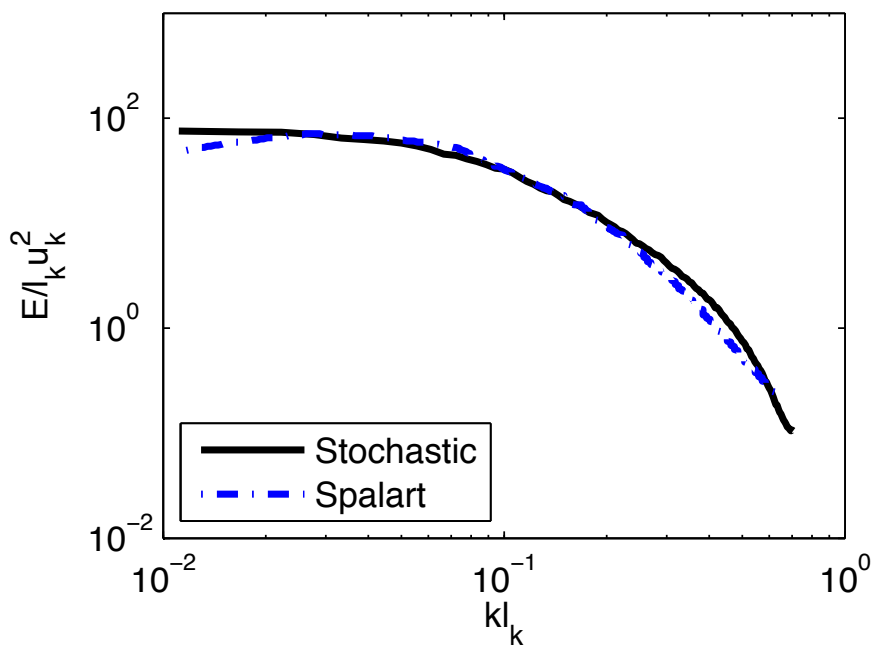

Figure 2: Turbulent kinetic energy spectra at $y+=100$ corresponding to the $v$ velocity component.

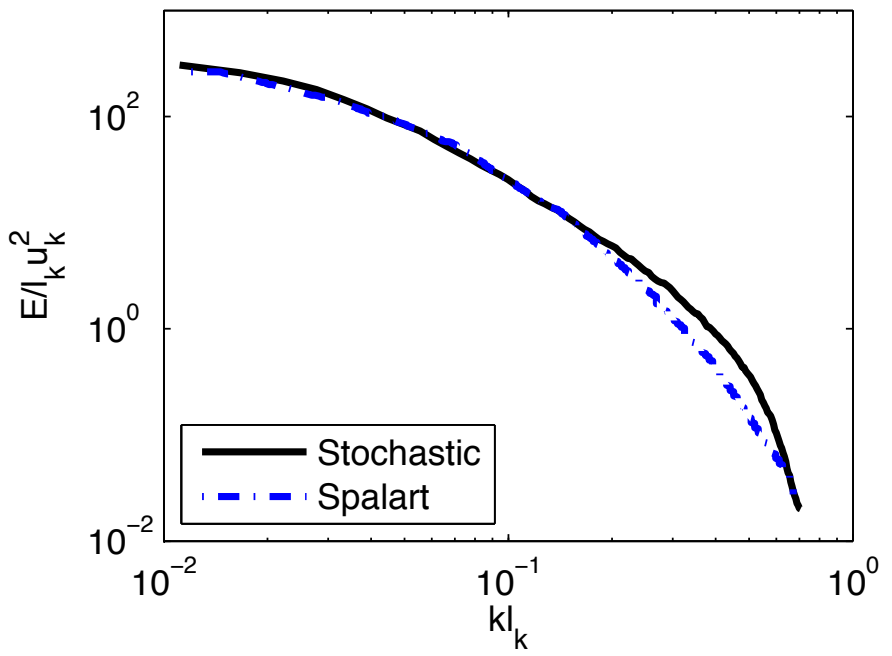

FiguRE 3: Turbulent kinetic energy spectra at $y+=100$ corresponding to the $w$ velocity component. 


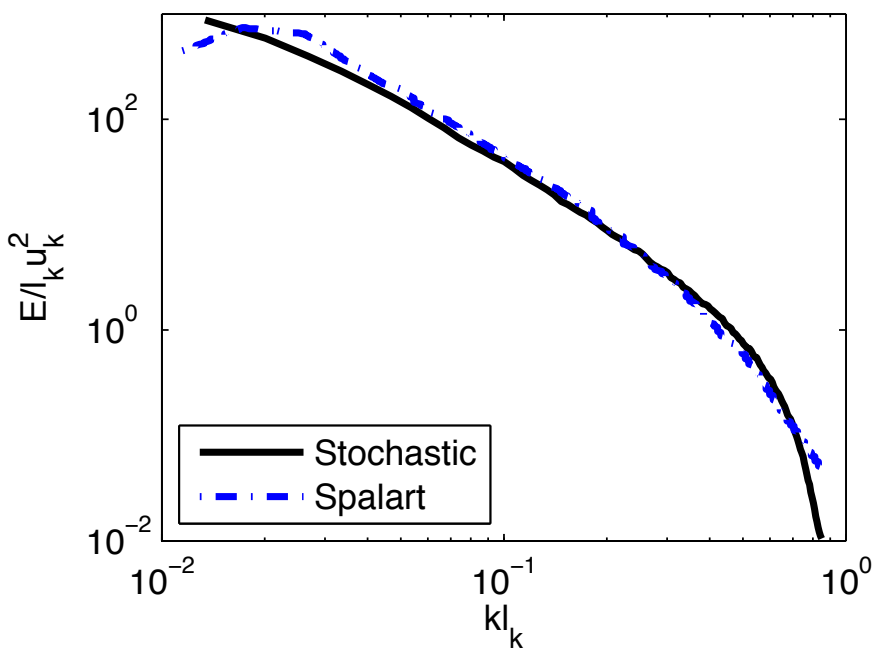

FIgURE 4: Turbulent kinetic energy spectra at $y+=200$ corresponding to the $u$ velocity component. Stochastic results using the current method are compared with results of Spalart [19]. Kolmogorov velocity and length scales are $\mathfrak{u}_{\mathrm{k}}=0.0241$ and $\boldsymbol{l}_{\mathrm{k}}=6.2335 \times 10^{-4}$ respectively. 


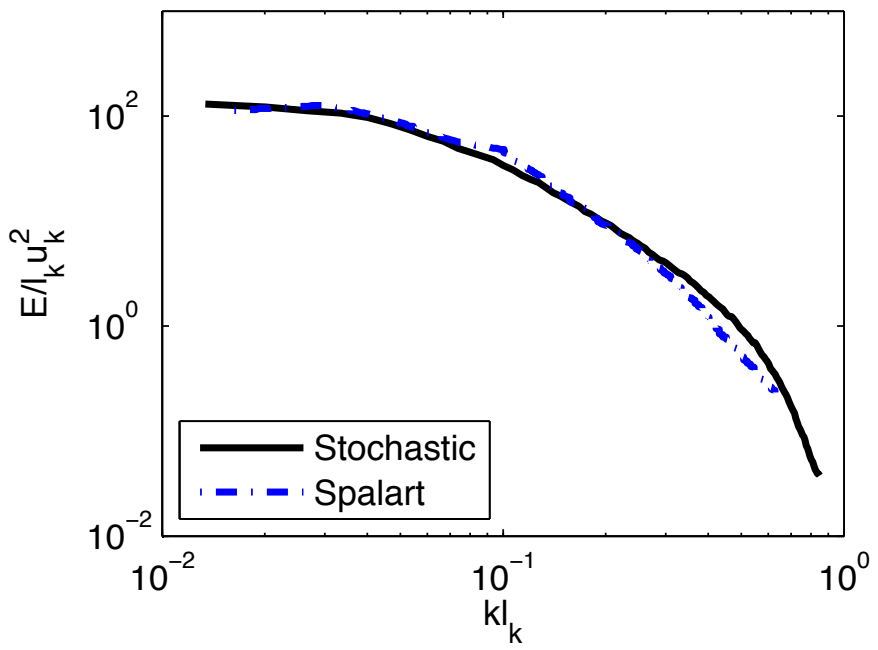

FiguRE 5: Turbulent kinetic energy spectra at $y+=200$ corresponding to the $v$ velocity component.

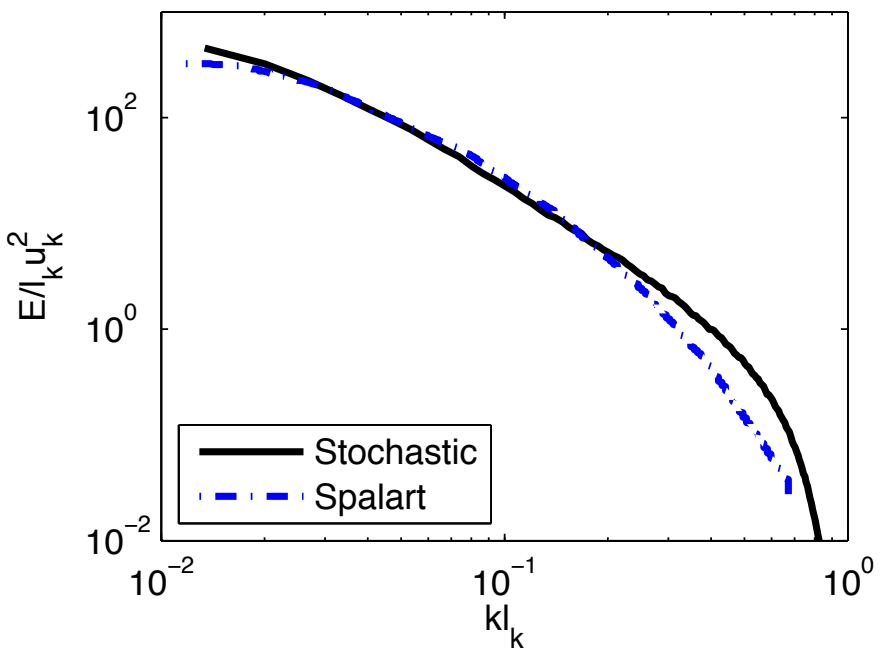

FiguRE 6: Turbulent kinetic energy spectra at $y+=200$ corresponding to the $w$ velocity components. 
spectra well once the model's tuning parameters were adjusted. Overall the most significant result of the present work is the insight into the importance of tuning the stochastic model to a particular turbulent boundary layer flow. To use the present technique for predicting turbulent noise, we recommend first obtaining reliable estimates of expected turbulent energy spectra and setting the appropriate tuning parameters before acoustic analysis begins.

\section{References}

[1] C. Bailly and D. Juve. Numerical solution of acoustic propagation problems using linearized euler equations. AIAA Journal, 38(1):22-29, 2000. doi:10.2514/2.949 C543, C545

[2] W. Bechara, C. Bailly, and Lafon. Stochastic approach to noise modeling for free turbulent flows. Journal of the Acoustical Society of America, 97(6):3518-3531, 1994. doi:10.2514/3.12008 C543

[3] M. Billson, L. Eriksson, and Davidson. Jet noise prediction using stochastic turbulence Modeling. 9th AIAA/CEAS Aeroacoustics Conference, (2003-3282), 2003. C543, C544, C545, C546

[4] C. Blom, B. Verhaar, and Van Der Heijden. A linearized euler method based prediction of turbulence induced noise using time averaged flow properties. AIAA Journal, (A01-16882), 2001. C543

[5] R. Ewert. Slat noise trend prediction using CAA with stochastic sound sources from a random particle-mesh method (RPM). AIAA Journal, (2006-2667), 2006. C543

[6] R. Ewert. RPM-the fast random particle-mesh method to realize unsteady turbulent sound sources and velocity fields for CAA applications. AIAA Journal, (2007-3506), 2007. C543 
[7] R. Ewert. Broadband slat noise prediction based on CAA and stochastic sound sources from a random particle-mesh (RPM) method. Computers and Fluids, 37(4):369-387, 2008. doi:10.1016/j.compfluid.2007.02.003 C543

[8] R. Ewert, C. Appel, and Dierke. RANS/CAA based prediction of NACA 0012 broadband trailing edge noise and experimental validation. 15th AIAA/CEAS Aeroacoustics Conference, 2009. C543, C545

[9] R. Ewert and W. Schroder. On the simulation of trailing edge noise with a hybrid LES/APE method. Journal of Sound and Vibration, 270:509-524, 2004. doi:10.1016/j.jsv.2003.09.047 C543, C544

[10] R. Ewert and R. Edmunds. CAA slat noise studies applying stochastic sound source based solenoidal digital filters. 26th AIAA Aeroacoustics Conference, 2005. C543

[11] M. Karweit, P. Belanc-Benon, and Juve. Simulation of the propagation of an acoustic wave through a turbulent velocity field: a study of phase variance. Journal of the Acoustical Society of America, 89(1):52-62, 1991. doi:10.1121/1.400415 C543

[12] M. Klein, A. Sdiki, and Janicka. A digital filter based generation of inflow data for spatially developing direct numerical or large eddy simulations. Journal of Computational Physics, 186:652-665, 2003. doi:10.1016/S0021-9991(03)00090-1 C543, C545

[13] R. H. Kraichnan. Diffusion by a random velocity field. The Physics of Fluids, 13:22-31, 1970. doi:10.1063/1.1692799 C543

[14] T. Lund, X. Wu, and Squires. Generation of turbulent inflow data for spatially developing boundary layer simulations. Journal of Computational Physics, 140:233-258, 1998. doi:10.1006/jcph.1998.5882 C543, C544 
[15] M. Mesbah. Flow noise prediction using the stochastic noise generation and radiation approach. Phd, Katholieke Universiteit Leuven, Faculteit Ingenieurswetenschappen, 2006. C543, C544, C546

[16] S. Chong, M. S. Perry, A. E. Henbest. A theoretical and experimental study of wall turbulence. Journal of Fluid Mechanics, 165:163-199, 1986. C547

[17] A. Smirnov, S. Shi, and Celik. Random flow generation technique for large eddy simulations and particle dynamics modeling. Journal of Fluids Engineering, 123:359-371, 2001. doi:10.1115/1.1369598 C543

[18] M. Snellen, L. Lier, and Golliard. Predictions of the flow induced noise for practical applications using the SNGR method. 10th International Congress on Sound and Vibration, 2003. C543

[19] P. R. Spalart. Direct simulation of a turbulent boundary layer up to $\operatorname{Re}(\theta)=1410$. Journal of Fluid Mechanics, 187:61-98, 1988. C546, C547, C548, C550

\section{Author addresses}

1. R. F. Jones, School of Mechanical Engineering, University of Adelaide, South Australia, Australia. mailto:richard.jones@adelaide.edu.au

2. C. J. Doolan, School of Mechanical Engineering, University of Adelaide, South Australia, Australia.

3. M. D. Teubner, School of Mathematical Sciences, University of Adelaide, South Australia, Australia. 